\title{
Understanding the stepwise mechanism in the formation of halogen-bonded organic cocrystals by mechanochemistry
}

Filip Topić, ${ }^{1}$ Poppy Hindle, ${ }^{2}$ Mihails Arhangelskis, ${ }^{1}$ Ricky $\operatorname{Tran},{ }^{1}$ Tomislav Friščić ${ }^{1}$

${ }^{1}$ Department of Chemistry, McGill University, 801 Sherbrooke Street W., Montreal, H3A OB8, Canada.

E-mail: filip.topic@mcgill.ca, tomislav.friscic@mcgill.ca

${ }^{2}$ School of Chemistry, Cardiff University, Main Building, Park Place, Cardiff, CF10 3AT, United Kingdom.

Mechanochemical synthesis of halogen-bonded cocrystals involving di- or multi-topic halogen bond donors and receptors has been shown to yield cocrystals of different stoichiometries, depending on their stoichiometric ratio, which can determine whether all or only some of the donor/acceptor functionalities are engaged in halogen bonding. For example, cocrystallization of a ditopic donor and a ditopic receptor might produce cocrystals with either 2:1 (all donor and half of the acceptor functionalities engaged), 1:1 (all donor and acceptor functionalities engaged) or 1:2 (half of the donor and all the acceptor functionalities engaged) donor/acceptor stoichiometries. Moreover, cocrystals of different stoichiometries could reasonably be expected to sometimes produce more than one polymorph, adding to the diversity of solid phases that can arise from a given donor-acceptor combination.

In order to better understand the factors guiding the mechanochemical formation of such stoichiometrically diverse cocrystal systems, we focused on several perfluorinated, bromine- or iodine-based halogen bond donors and nitrogen-based acceptors. Mechanochemical experiments were performed by neat or liquid-assisted grinding, with different grinding durations and employing different stoichiometric ratios of donors and acceptors. The phases appearing in mechanochemical cocrystallization were characterized by powder X-ray diffraction, with single-crystal X-ray diffraction used to elucidate novel structures. Plane-wave periodic DFT calculations were used to evaluate the energies of different cocrystal phases and elucidate the thermodynamic aspects of cocrystal stoichiometric transformations.

Our systematic studies of mechanochemical cocrystallization reveal not only that the formation of the final product is determined by reactant stoichiometric ratio, but also that it involves the appearance of one or more intermediate phases. The intermediates in this stepwise mechanochemical process were found to be cocrystals with different stoichiometric compositions and/or different polymorphs. The appearance of these intermediates, and their sequence in the stepwise reaction mechanism, was subsequently explained through crystal structure analysis accompanied by the computational evaluation of thermodynamic enthalpic stability. 